\title{
SMALL VOLUME ON BIG $n$-SPHERES
}

\author{
CHRISTOPHER B. CROKE
}

(Communicated by Jon G. Wolfson)

\begin{abstract}
We consider Riemannian metrics on the $n$-sphere for $n \geq 3$ such that the distance between any pair of antipodal points is bounded below by 1 . We show that the volume can be arbitrarily small. This is in contrast to the 2-dimensional case where Berger has shown that Area $\geq 1 / 2$.
\end{abstract}

In 1977, Berger [B77] considered the set of Riemannian metrics $g$ on the $n$-sphere such that $d(x, A(x)) \geq 1$ and defined the constants:

$$
h(n) \equiv \inf \{\operatorname{Vol}(g)\} .
$$

We will also consider $\bar{h}(n) \equiv \inf \left\{\operatorname{Vol}(g) \mid A^{*} g=g\right\}$, where you can think of $A$ as the standard antipodal map or simply set $A$ to be any fixed point free diffeomorphism of $S^{n}$ with $A^{2}=I d$.

One of Berger's reasons for his interest in these invariants was their use in getting lower bounds on the volume of balls $B(p, R)$ in a complete Riemannian manifold with $R$ less than half the injectivity radius $\operatorname{inj}(M)$. In this case all geodesics in the ball minimize distance. Hence for all $r \leq R$ the distance in $B(p, R)$ between antipodal points of $S(p, r)=\partial B(p, r)$ is $2 r$, and thus the intrinsic distance in $S(p, r)$ is even larger. So we get $\operatorname{Vol}(S(p, r)) \geq h(n-1)(2 r)^{n-1}$ and

$$
\begin{aligned}
\operatorname{Vol}(B(p, R)) & =\int_{0}^{R} \operatorname{Vol}(S(p, r)) d r \\
& \geq \int_{0}^{R} h(n-1)(2 r)^{n-1} d r=2^{n-1} \frac{h(n-1)}{n} R^{n} .
\end{aligned}
$$

Berger noted that $h(1)=2$ and proved $h(2) \geq \frac{1}{2}$ to get a (nonsharp) lower bound $\operatorname{Vol}(B(p, R)) \geq 2 R^{2}$ on the volume of 2 -balls and $\operatorname{Vol}(B(p, R)) \geq \frac{2}{3} R^{3}$ for 3 -balls when $R \leq \operatorname{inj}(M) / 2$.

In this short note we show by relatively easy examples:

Theorem 0.1. $h(n)=0$ for $n \geq 3$.

This question and some history surrounding it was also discussed in section 6 of [CK03]. In particular, Ivanov (see [I98]) has given examples of a sequence of metrics on $S^{3}$ that Gromov-Hausdorff converge to the standard metric but whose volumes go to zero.

Received by the editors November 9, 2006.

2000 Mathematics Subject Classification. Primary 53C20.

The author was supported by NSF grants DMS 02-02536 and 07-04145.

(C) 2007 American Mathematical Society Reverts to public domain 28 years from publication 
The main open question about volume of balls can be stated as a conjecture which is open in all dimensions (even $n=2$ !).

Conjecture 0.2. If $\omega_{n}$ represents the volume of the unit $n$-sphere:

a) For any $r \leq \frac{\operatorname{inj}(M)}{2}, \operatorname{Vol}(B(r)) \geq \frac{\omega_{n}}{2}\left(\frac{2}{\pi}\right)^{n} r^{n}$ with equality holding only if the ball is isometric to a hemisphere.

b) For any $r \leq \operatorname{inj}(M), \operatorname{Vol}(B(r)) \geq \frac{\omega_{n}}{\pi^{n}} r^{n}$, where equality holds if and only if $M$ is isometric to the round sphere of injectivity radius $r$ (i.e. extrinsic radius $\frac{r}{\pi}$ ).

As mentioned above, Berger [B77] gave nonsharp lower bounds for $n=2$ and $n=3$. The author gave nonsharp lower bounds in all dimensions in [Cr80] and showed that $b$ ) was true for the "average" ball (i.e. $\frac{1}{\operatorname{Vol}(M)} \int_{M} \operatorname{Vol}(B(x, r)) d x \geq$ $\left.\frac{\omega_{n}}{\pi^{n}} r^{n}\right)$ for any compact manifold $M$ in any dimension [Cr84]. This followed Berger's isoembolic inequality $\operatorname{Vol}(M) \geq \frac{\omega_{n}}{\pi^{n}} \operatorname{inj}(M)^{n}$, where equality holds if and only if $M$ is isometric to the round sphere.

As to the question of the exact value of $h(2)$ we have the following interesting

Conjecture 0.3. $h(2)=\frac{4}{\pi}$, and the round sphere is the only space that achieves this.

The situation for $\bar{h}$ is quite different. By identifying antipodal points we get a metric on $\mathbb{R} P^{n}$ such that all noncontractible closed curves have length at least 1 (i.e. $\operatorname{sys}(M) \geq 1$ ). Hence Pu's Theorem [Pu52] shows $\bar{h}(2)=\frac{4}{\pi}$, while Gromov's theorem [Gr83] gives us nonsharp positive lower bounds for $\bar{h}(n)$ for all $n$. The question as to the actual values of $\bar{h}(n)$ for $n \geq 3$ is a hard and very interesting one.

One other place where this notion came up is in [Cr02]. There it is shown that for a Riemannian metric on a 3-sphere with $d(x, A(x)) \geq D$ and $L=$ length of the shortest closed geodesic, we have $\mathrm{Vol}^{1 / 3} \geq$ const $\min \{L, 2 D\}$. In this note we show that there is no constant such that $\mathrm{Vol}^{1 / 3} \geq$ constD. It is still an open (and very interesting) question if there is a constant so that $\mathrm{Vol}^{1 / 3} \geq$ const L.

We now construct the examples on the 3 -sphere.

We will let $M B_{\epsilon}$ be the $\epsilon$ neighborhood in the flat plane $\mathbb{R}^{2}$ of a tripod (three unit length line segments from the origin making angles $\frac{2 \pi}{3}$ with each other). As $\epsilon$ goes to 0 the area goes to 0 while the length of the boundary goes to 6 . This has been known for a while as an example of a metric on a 2-disc whose area goes to 0 but such that on the boundary antipodal points satisfy $d_{M B_{\epsilon}}\left(p, A_{\epsilon}(p)\right) \geq d_{\mathbb{R}^{2}}\left(p, A_{\epsilon}(p) \geq 1\right.$. Here $A_{\epsilon}(p)$ is the point half way around the boundary. (Note that the nonconvexity of $M B_{\epsilon}$ is not the point here since one could use equilateral triangles in a more and more negatively curved simply connected space form.)

Our examples are just $M_{\epsilon}^{3}=\partial\left(M B_{\epsilon} \times M B_{\epsilon}\right)$, which as the boundary of a 4ball is a 3 -sphere. Since $M_{\epsilon}=\left(\partial\left(M B_{\epsilon}\right) \times M B_{\epsilon}\right) \cup\left(M B_{\epsilon} \times \partial\left(M B_{\epsilon}\right)\right), \operatorname{Vol}\left(M_{\epsilon}\right)=$ $2 \operatorname{Area}\left(M B_{\epsilon}\right) L\left(\partial\left(M B_{\epsilon}\right)\right)$. Hence $\lim _{\epsilon \rightarrow 0} \operatorname{Vol}\left(M_{\epsilon}\right)=0$.

We need to define the antipodal map $\bar{A}_{\epsilon}$ on $M_{\epsilon}$. First extend $A_{\epsilon}$ to a continuous homeomorphism $A_{\epsilon}: M B_{\epsilon} \rightarrow M B_{\epsilon}$ with a single fixed point (the origin in $\mathbb{R}^{2}$ say). One can do this simply by mapping the line segment between the origin and $p \in \partial\left(M B_{\epsilon}\right)$ to the line segment between the origin and $A_{\epsilon}(p)$. Then let

$$
\bar{A}_{\epsilon}=\left.\left(A_{\epsilon} \times A_{\epsilon}\right)\right|_{M_{\epsilon}} .
$$


For a point $(p, q) \in \partial\left(M B_{\epsilon}\right) \times M B_{\epsilon} \subset M_{\epsilon}$ we have

$$
\begin{aligned}
d_{M_{\epsilon}}\left((p, q), \bar{A}_{\epsilon}(p, q)\right) & =d_{M_{\epsilon}}\left((p, q),\left(A_{\epsilon}(p), A_{\epsilon}(q)\right)\right) \\
& \geq d_{\mathbb{R}^{4}}\left((p, q),\left(A_{\epsilon}(p), A_{\epsilon}(q)\right)\right) \geq d_{\mathbb{R}^{2}}\left(p, A_{\epsilon}(p)\right) \geq 1 .
\end{aligned}
$$

Similarly if $(p, q) \in M B_{\epsilon} \times \partial\left(M B_{\epsilon}\right) \subset M_{\epsilon}$, then $d_{M_{\epsilon}}\left((p, q), \bar{A}_{\epsilon}(p, q)\right) \geq 1$.

This completes the proof of the theorem in the 3 -dimensional case after smoothing the metric and $\bar{A}_{\epsilon}$. The higher-dimensional cases are handled by spherical suspension. Specifically given a metric $g$ on the $n$-sphere $S^{n}$ we construct a (singular) warped product metric $g^{\prime}=\cos ^{2}(t) g+d t^{2}$ on the $(n+1)$-sphere $S^{n+1}=$ $\left(S^{n} \times\left[-\frac{\pi}{2}, \frac{\pi}{2}\right]\right) / \sim$, where $\left(x, \frac{\pi}{2}\right) \sim\left(y, \frac{\pi}{2}\right)$ and $\left(x,-\frac{\pi}{2}\right) \sim\left(y,-\frac{\pi}{2}\right)$ for all $x, y \in S^{n}$. The antipodal map $A_{n+1}: S^{n+1} \rightarrow S^{n+1}$ is just $A_{n+1}(x, t)=\left(A_{n}(x),-t\right)$. It is easy to check that if $g$ has $d\left(x, A_{n}(x)\right) \geq \pi$ and $\operatorname{Vol}(g)=V$, then $D\left(x, A_{n+1}(x)\right) \geq \pi$ and $\operatorname{Vol}\left(g^{\prime}\right)=\operatorname{Vol}(g) \int_{-\frac{\pi}{2}}^{\frac{\pi}{2}} \cos ^{n}(t) d t=\frac{\omega_{n+1}}{\omega_{n}} \operatorname{Vol}(g)$. So spherically suspending (and smoothing) a sequence of examples that shows $h(n)=0$ gives us a sequence showing that $h(n+1)=0$.

\section{REFERENCES}

[B77] M. Berger, Volume et rayon d'injectivité dans les variétés riemanniennes de dimension 3, Osaka J. Math., 14 (1977), 191-200. MR0467595 (57:7451)

[Cr80] C. Croke, Some isoperimetric inequalities and eigenvalue estimates, Ann. Scient. Ec. Norm. Sup., 4e série, t.13 (1980), 419-435. MR608287 (83d:58068)

[Cr84] C. Croke, Curvature Free Volume Estimates, Inventiones Mathematicae 76 (1984), 515521. MR746540 (85f:53044)

[Cr02] C. Croke, The volume and lengths on a three sphere, Comm. Anal. Geom., 10 (2002) no. 3, 467-474. MR1912255 (2003c:53041)

[CK03] Croke, C.; Katz, M., Universal volume bounds in Riemannian manifolds, Surveys in Differential Geometry VIII, Lectures on Geometry and Topology held in honor of Calabi, Lawson, Siu, and Uhlenbeck at Harvard University, May 3-5, 2002, edited by S.T. Yau (Somerville, MA: International Press, 2003.) pp. 109-137. See arXiv:math.DG/0302248 MR2039987 (2005d:53061)

[Gr83] Gromov, M., Filling Riemannian manifolds, J. Diff. Geom. 18 (1983), 1-147. MR697984 (85h:53029)

[198] S. Ivanov, Gromov-Hausdorff Convergence and volumes of manifolds, St. Petersburg Math. J., 9(1998) No.5, 945-959 MR1604389 (98k:53052)

[Pu52] Pu, P.M., Some inequalities in certain nonorientable Riemannian manifolds, Pacific J. Math. 2 (1952), 55-71. MR0048886 (14:87e)

Department of Mathematics, University of Pennsylvania, Philadelphia, Pennsylvania 19104-6395

E-mail address: ccroke@math.upenn.edu 